\title{
Irreversible Pulmonary Hypertension Associated with the use of Interferon Alpha for Chronic Hepatitis C
}

\author{
Sonu Dhillon • Anshul Kaker • Aneil Dosanjh • \\ Deepa Japra $\cdot$ David H. VanThiel
}

Received: 27 October 2009/Accepted: 23 March 2010/Published online: 22 April 2010

(C) The Author(s) 2010. This article is published with open access at Springerlink.com

\begin{abstract}
The interferons are a complex group of virally induced proteins produced by activated macrophages and lymphocytes, which have become the mainstay of therapy for hepatitis C infection. Sustained viral response (SVR) rates in noncirrhotic patients vary from $40-80 \%$ with interferon-based therapy. This, along with transplantation, has drastically changed the course of hepatitis $\mathrm{C}$ virus (HCV) infection over the last two decades. Numerous side effects associated with interferon therapy have been reported. These range from transient flu-like symptoms to serious effects such as cardiac arrhythmias, cardiomyopathy, renal and liver failure, polyneuropathy, and myelosuppression. Pulmonary side effects including pneumonitis, pulmonary fibrosis, and reversible pulmonary hypertension have been reported. Herein, we present four cases in which irreversible pulmonary hypertension was diagnosed after prolonged treatment with interferon alpha. In each case, other causes of pulmonary hypertension were systematically eliminated. Pulmonary artery hypertension, which may be irreversible, should be considered in patients being treated with interferon alpha who present with exertional dyspnea and do not have a readily identifiable inflammatory or thromboembolic cause.
\end{abstract}

S. Dhillon $(\varangle) \cdot$ D. H. VanThiel

Department of Medicine, Rush University Medical Center,

1725 W. Harrison St., Ste. 158 Professional Building, Chicago,

IL 60612, USA

e-mail: sd7441@gmail.com

A. Dosanjh

University of California, Davis, CA, USA

A. Kaker · D. Japra

Pacific Cardiology Associates, Fremont, CA, USA
Keywords $\mathrm{HCV} \cdot$ Interferon alpha · Pulmonary hypertension · Liver · Lung

\section{Introduction}

The interferons are a complex group of virally induced host proteins produced by activated macrophages and lymphocytes. They were first described in 1957 by Isaacs and Lindenmann [1]. Alpha, beta, and gamma interferons have been identified and characterized. Interferon alpha (IFN $\alpha$ ) has been used extensively in the treatment of hepatitis viruses, as well as a few hematologic, nephrologic, and dermatologic malignancies. Although the exact mechanism of action of IFN $\alpha$ remains unclear, its usefulness in clinical practice is well established. It is most commonly used to treat chronic hepatitis $\mathrm{C}$ virus (HCV), either as monotherapy or in conjunction with ribavirin. In the past, the dose used for this purpose has ranged from 3 to 5 million units daily with $800-1,200 \mathrm{mg} /$ day of oral ribavirin for 1 year [2]. Two pegylated IFN $\alpha$ preparations enabling weekly dosing are now available and, along with ribavirin, are the current standard of care. A biosynthetic alpha interferon, consensus IFN (cIFN), is also available.

A sustained viral response (SVR) rate is defined as clearance of virus after 6 months following completion of active treatment. SVR rates currently vary from $40-80 \%$ in noncirrhotic patients depending upon the viral load, genotype, dosage, and formulation of IFN $\alpha$ utilized [2]. IFN $\alpha$ has also been used in the treatment of chronic myelogenous leukemia (CML), multiple myeloma, and renal cell carcinoma. The doses used for these nonhepatic diseases, especially CML, are much higher than those used for the treatment of HCV. Typical dosages range from 10 to 20 million units/day for 12-18 months. Nearly a third of 
IFN $\alpha$-treated patients with CML achieve complete cytologic remission and often remain disease-free for years $[3,4]$.

Numerous side effects have been reported as a consequence of interferon therapy. Almost all patients experience transient flu-like symptoms with fever, weakness, myalgias, headache, and tachycardia. These symptoms typically subside over the next 24 hours and are less prominent with treatment continuation. More serious effects include cardiac arrhythmias, cardiomyopathy, polyneuropathy, and myelosuppression, as well as liver and renal failure. Furthermore, various autoimmune diseases such as psoriasis, sprue, diabetes mellitus, thyroid disorders, and various forms of arthritis can be exacerbated or precipitated by IFN $\alpha$ therapy [5]. Pulmonary side effects include pneumonitis, pulmonary fibrosis, and a single report of new onset pulmonary hypertension [6, 7]. Resolution of these numerous untoward effects with discontinuation of the agent has been the rule.

Herein is reported a series of four individuals, who developed an irreversible, progressive, and severe form of pulmonary hypertension during treatment with IFN $\alpha$ for chronic HCV. This complication has not been reported previously and should be considered as a rare but important adverse effect of IFN $\alpha$ therapy.

\section{Case Reports}

\section{Case No. 1}

GB was a 35-year-old white male who initially presented with lower extremity edema and weakness and was found to have end-stage liver disease due to HCV and alcohol abuse. Over the next several months, he developed decompensated liver disease with ascites. His past medical history was significant for a cholecystectomy for gallstones. His family history was significant for a mother who died of lung cancer and a father with coronary artery disease. He started drinking excessively at the age of 16 and occasionally used other illegal drugs, including amphetamines, heroin, and intranasal cocaine. He abstained from drug and alcohol use from the time of his initial presentation.

Physical examination was significant for a nontender but distended abdomen. The liver edge was $3 \mathrm{~cm}$ below the costal margin and a palpable spleen tip was present. Shifting dullness was present and pedal edema was noted to the level of the knees. The lungs were clear bilaterally. Cardiac examination revealed a normal rate and rhythm with no murmurs or gallop sounds.

Initial laboratory evaluation revealed normal electrolytes, normal renal function, and a prothrombin time of
$15.8 \mathrm{~s}$ (normal 10.8-12.8 s). Serum aminotransferase levels (AST/ALT) were 226/183 IU/L, with a normal alkaline phosphatase and total bilirubin level. The serum albumin level was $3.3 \mathrm{mg} / \mathrm{dl}$. A complete blood count documented a platelet count of $89,000 / \mathrm{mm}^{3}$. Serum tests were positive for HCV-RNA genotype 1a infection with a viral load $>$ $1 \times 10^{6}$ copies $/ \mathrm{mL}$.

An abdominal computed tomography scan revealed a nodular cirrhotic liver with a volume of $1,600 \mathrm{~cm}^{3}$. The presence of prominent perigastric varices and periumbilical collaterals consistent with portal hypertension were noted also. Abdominal ultrasound documented the liver to have increased echogenicity. The portal and hepatic veins as well as the hepatic arteries were patent. A echocardiogram documented a left ventricular ejection fraction of $60 \%$ with normal heart valves and a normal estimated pulmonary artery pressure (PAP).

A liver biopsy was obtained and interpreted as showing grade 4 , stage 4 disease. He was started on IFN $\alpha 3$ million units daily in May 1999 in an effort to clear his viremia prior to transplantation. His viral load rapidly decreased and became undetectable at 8 weeks. He underwent orthotopic liver transplant 6 months later.

Postoperatively, he was found to have recurrent $\mathrm{HCV}$ with a viral load of $8.5 \times 10^{5}$ copies $/ \mathrm{mL}$. He was started on cIFN at a dose of $15 \mathrm{ug} /$ day. He again responded rapidly and was $\mathrm{HCV}$ polymerase chain reaction (PCR)-negative by 8 weeks. His only complaint during the treatment period was persistent fatigue, which was attributed to the IFN $\alpha$ therapy.

After approximately 1 year of therapy, he began to experience progressive dyspnea on exertion, fatigue, and edema. An examination at this time revealed a resting tachycardia; a new grade III/VI systolic ejection murmur which increased in intensity with inspiration. He was admitted to hospital for further evaluation and was found to have severe pulmonary hypertension with right heart failure.

Cardiac catheterization revealed PAP of $81 / 30 \mathrm{mmHg}$, a cardiac output of $8.33 \mathrm{~L} / \mathrm{min}$, pulmonary vascular resistance (PVR) of $8.26 \mathrm{~cm} \mathrm{H}_{2} \mathrm{O} / \mathrm{L} / \mathrm{min}$, and a cardiac index of $2.61 \mathrm{~L} / \mathrm{min} / \mathrm{m}^{2}$. He experienced no change in his symptoms with the use of short-acting vasodilators. A ventilation perfusion scan was reported as having low probability for pulmonary emboli. A diagnosis of pulmonary hypertension of unknown etiology was made. He was classified as NYHC III and was started on bosentan, an endothelin receptor blocker, as well as a calcium channel blocker, a diuretic, and digitalis.

$\mathrm{He}$ failed bosentan as well as treprostinil, a prostacyclin analog, but had a minor response to epoprostenol. He required repeated admissions for progressive cardiac decompensation and the development of a persistent right- 
sided pleural effusion. Ultimately, he died in November 2002. The family refused an autopsy.

\section{Case No. 2}

DS was a 40-year-old female who was found to have an $\mathrm{HCV}$ infection while attempting to donate blood. Her past medical history included mild asthma, depression, a panic disorder, and multiple orthopedic surgeries related to a motor vehicle accident 20 years earlier, at which time she received 3 units of packed red blood cells. Her only medication was an inhaled bronchodilator. Social history included a 30-pack-year smoking history with occasional alcohol use. No intravenous drug use was reported.

A review of systems was unremarkable. Physical examination revealed clear lungs, and cardiac examination was with regular heart rate and no murmur or gallop. Abdominal examination revealed normal bowel sounds, no tenderness, and no hepatosplenomegaly. No pedal edema was appreciated. Laboratory examination included normal electrolytes, renal function, and liver function tests. The patient was found to have a genotype $1 \mathrm{~b}$ infection with a viral load of $1.2 \times 10^{6}$ copies $/ \mathrm{mL}$. A liver biopsy was obtained and interpreted as showing grade 1 stage 1 disease. Mild steatosis was noted also. An upper gastrointestinal endoscopy was unremarkable. She was started on IFN $\alpha$ therapy ( 3 million units daily) with ribavirin ( $800 \mathrm{mg}$ daily). The ribavirin was discontinued after 4 months because of profound anemia ( $\mathrm{Hgb} 4.5 \mathrm{gm} / \mathrm{dL}$ ). She failed to achieve viral clearance, despite being on maintenance therapy for 32 months.

In February 2001, she reported progressive dyspnea on exertion, edema, and weight gain. This required hospitalization and the IFN $\alpha$ therapy was discontinued. On physical examination, an elevated jugulo-venous pressure was appreciated, the lungs were clear, and cardiac examination revealed a grade $2 / 6$ systolic ejection murmur at the left lower sternal border with a prominent pulmonic second sound that increased with inspiration. Pedal edema was present up to her knees. Neurologic examination was unremarkable.

An EKG showed sinus rhythm with voltage criteria for right ventricular hypertrophy and left atrial enlargement. Her chest X-ray was unremarkable. Pulmonary function studies were consistent with minimal asthma. She had unremarkable arterial blood gas on room air ( $\mathrm{pH}$ 7.42, $\mathrm{pO}_{2}$ 102, $\mathrm{pCO}_{2}$ 33). A cardiac echocardiogram demonstrated normal left ventricular ejection fraction (59\%), diffusely dilated, hypertrophied, and hypokinetic right ventricle, severe tricuspid regurgitation, and a small pericardial effusion without evidence of cardiac tamponade. A ventilation perfusion scan was interpreted as having an intermediate probability of pulmonary embolism. A subsequent pulmonary angiogram revealed no evidence of thromboembolic disease. Right heart catheterization documented a right atrial pressure of $24 \mathrm{~mm} \mathrm{Hg}$, right ventricle systolic pressure of $67 \mathrm{~mm} \mathrm{Hg}$, right ventricle end diastolic pressure of $11 \mathrm{~mm} \mathrm{Hg}$, PAP of $71 / 34 \mathrm{~mm} \mathrm{Hg}$ with a mean pulmonary arterial pressure of $52 \mathrm{~mm} \mathrm{Hg}$, and pulmonary artery wedge pressure of $24 \mathrm{~mm} \mathrm{Hg}$.

Severe pulmonary hypertension with NYHC III heart failure of uncertain etiology was diagnosed. She was started on a diuretic and a calcium channel blocker and experienced a gradual worsening of her symptoms. Subsequently, she was started on treprostinil, with stabilization but no reversal of her pulmonary hypertension.

Case No. 3

FM was a 50-year-old male who presented initially with a pericardial effusion in 1997. At that time, the effusion was drained, with no specific etiology being identified, although he was known to be HCV-positive. Subsequent workup revealed advanced liver disease with decompensated cirrhosis. He underwent liver transplantation soon thereafter. The post-transplant course was uncomplicated, except for recurrent HCV. His past medical history was significant for obstructive sleep apnea, hyperthyroidism, and hypertriglyceridemia. Social history revealed 40-pack-year tobacco use, which he terminated approximately 10 years earlier, and a distant history of alcohol and cocaine use. He was married and worked in a soft drink bottling factory. A review of systems was noncontributory. Physical examination revealed an obese man in no distress. The lungs were clear to auscultation bilaterally and the cardiac examination was unremarkable. The abdomen was protuberant and nontender with palpable hepatosplenomegaly. No ascites was appreciated and he had no edema.

The laboratory data was remarkable for mild renal insufficiency (BUN/Cr $23 \mathrm{mg} / \mathrm{dl} / 1.6 \mathrm{mg} / \mathrm{dl}$ ) with a platelet count of $105,000 / \mathrm{mm}^{3}$ and a WBC count of $4.1 \times 1,000$ cells $/ \mathrm{mm}^{3}$. Liver function tests were normal and he was found to have an HCV viral load of $2.5 \times 10^{5}$ copies $/ \mathrm{mL}$. A liver biopsy showed stage I disease with no portal or lobular inflammation and mild sinusoidal fibrosis.

IFN $\alpha$ therapy (5 million units/day) with ribavirin $200 \mathrm{mg}$ BID was initiated. After being on therapy for 10 months, he developed progressive dyspnea on exertion with pedal edema. The patient was admitted to hospital and the IFN $\alpha$ and ribavirin were discontinued. Physical examination showed him to have clear lungs, and a cardiac examination revealed an early systolic murmur with a prominent pulmonic second sound. The abdomen was protuberant with easily palpable hepatomegaly and normoactive bowel sounds. Mild lower extremity edema was noted. 
His evaluation included pulmonary function testing, which showed a significant restrictive defect with dramatic reduction in diffusion capacity. Computed tomography of the abdomen showed massive hepatomegaly (liver volume $3,448 \mathrm{cc}^{3}$ ), splenomegaly, and ascites. A ventilation perfusion scan was negative for thromboembolic disease. An echocardiogram documented severe left ventricular hypertrophy, enlargement of the right heart chambers with severe tricuspid regurgitation, and an elevated PAP ( $80 \mathrm{~mm} \mathrm{Hg}$ ). A diagnosis of severe pulmonary hypertension with resultant cor pulmonale was made. The IFN therapy was discontinued and treatment was initiated with a diuretic and a calcium channel blocker with stabilization, but there was no improvement in his pulmonary hypertension.

Case No. 4

DM was a 49-year-old male who was noted to have elevated serum aminotransferase levels (AST/ALT 228/136 IU/L). Workup revealed chronic HCV infection. He was in good health and had no specific complaints. His past medical history was unremarkable. He had no allergies and was on no medications. Social history revealed a 40-packyear tobacco history and occasional use of alcohol. He denied any intravenous drug use. Family history was negative. A review of systems was positive only for generalized malaise. Physical examination revealed clear lungs, cardiac examination with no murmurs, the abdomen was soft with a liver and a spleen palpable 3-4 finger breadths below the costal margins. No peripheral edema was present.

Laboratory evaluation revealed normal electrolytes and renal function. Complete blood count showed WBC $2.8 \times 1,000$ cells $/ \mathrm{mm}^{3}$ and a platelet count of $80,000 /$ $\mathrm{mm}^{3}$. Liver function tests documented serum aminotransferase elevations with a normal bilirubin level. HCV genotype 1 was found with a viral load of $1.8 \times 10^{6}$ copies.

A computed tomography scan of the abdomen revealed a cirrhotic liver with splenomegaly and evidence of portal hypertension with recanalization of the periumbilical veins. An upper endoscopy revealed grade II esophageal varices with portal hypertensive gastropathy.

He was started on IFN $\alpha$ therapy (PEG-Intron ${ }^{\circledR} 2 \mu \mathrm{g} / \mathrm{kg}$ / week) with ribavirin (200 mg BID). Eight months after the initiation of therapy, the patient developed progressive dyspnea on exertion with increasing abdominal girth and pedal edema. The IFN $\alpha$ and ribavirin therapy were discontinued. Re-evaluation at this time revealed a normal chest X-ray. Pulmonary function tests documented a moderate to severe decrease in diffusion capacity with a mild reversible obstructive ventilatory defect. A cardiac echogram documented normal left ventricular size and function (EF 60\%) and a normal right ventricular size. Moderate tricuspid regurgitation was noted, with a markedly elevated PAP of $80 \mathrm{~mm} \mathrm{Hg}$. A ventilation perfusion scan was negative for thromboembolic disease. The patient was diagnosed as having severe pulmonary hypertension of unknown etiology and was treated with a diuretic and calcium channel blocker with good results. He remains clinically stable however, without improvement in his pulmonary hypertension.

\section{Discussion}

In these four cases, each individual reported a new symptom of respiratory insufficiency after prolonged therapy with IFN $\alpha$. Evaluation revealed pulmonary hypertension which was irreversible upon discontinuation of IFN $\alpha$ and with no identifiable etiology. There is no common variable other than HCV and IFN $\alpha$ therapy among these individuals. Three of four were noncirrhotic and 2/4 were postliver transplant with good hepatic function and uncomplicated postsurgical course. There was also no commonality in the preparation of the interferon used. The dosage and duration used were not unlike those used typically for hepatic or hematologic disease. The dosage of cIFN used was $15 \mu \mathrm{g}$ daily, which was higher than the approved $9 \mu \mathrm{g} /$ three times per week; however, this regimen is now commonly used. It was recently evaluated in a multicenter study, the DIRECT trial, with validation of its safety and efficacy in those who fail standard therapy [8]. Also, it is unlikely that an interaction with any other medications, such as immunosuppressants, is contributory to this finding, but such an association cannot be ruled out categorically.

Potential mechanisms that might explain this sequence of events might include a pre-existing condition, IFN $\alpha$ induced pulmonary hypertension, or the acceleration of a previously subclinical phenomenon caused by other factors such as human herpesvirus 8 (HHV8), HCV itself, or a previously unrecognized genetic predisposition.

The development of primary pulmonary hypertension secondary to portal hypertension, called portopulmonary hypertension, is a well-known entity [9]. Cirrhosis has been reported to increase the risk of pulmonary hypertension 6-20-fold [10]. This risk increases proportional to the duration of the portal hypertension, although there is no documented relationship to the severity of portal hypertension, degree of hepatic failure, or fraction of blood flow shunting through the lungs [10]. It is most often seen in patients with end-stage liver disease and is considered as one of several rare sequelae of prolonged portal hypertension. In the present series, only case 4 had evidence of significant portal hypertension prior to the 
initiation of therapy. Patients 1 and 3 had a liver transplant prior to the time of diagnosis and patient 2 had only mild hepatic fibrosis on biopsy. In each case, other causes of portal hypertension were pursued vigorously and systematically ruled out.

$\mathrm{HCV}$ is a well-known cause of cardiopulmonary pathology. Numerous reports have linked HCV to idiopathic pulmonary fibrosis [11, 12], pneumonitis [13], and cardiomyopathy [14]. In this setting, pulmonary hypertension is secondary to end-stage pulmonary fibrosis, which is easily recognized with standard radiologic evaluations. In this series, no evidence for the presence of pulmonary fibrosis was present in any of the four cases.

Thus, this series of cases suggests that an association between pulmonary hypertension and IFN $\alpha$ therapy may exist. IFN $\alpha$ is a powerful immunomodulator that has a variety of effects on the lungs. Though considered uncommon, most reported cases of adverse pulmonary side effects related to IFN $\alpha$ consist of an exacerbation of asthma [15], pleural effusion [16], an activation of sarcoidosis [17], bronchiolitis obliterans-organizing pneumonia [18], and bilateral pulmonary infiltrates and reversible pulmonary hypertension. Several reports have described the phenomenon of bilateral interstitial and alveolar infiltrates, which have been shown histologically to be interstitial pneumonitis [19-21]. In contrast, there is only one reported case of pulmonary hypertension which was reversible, having developed in a patient receiving IFN $\alpha$ for CML [7].

An association between IFN $\alpha$ and pulmonary hypertension has been described in sheep. Hanaoka et al. found that, $1 \mathrm{~h}$ after an infusion of IFN $\alpha$ directly into the lungs of sheep, PAP and PVR increased significantly and these events coincided with elevations of thromboxane $\mathrm{B} 2$ in the plasma. Furthermore, the administration of OKY-046, a selective thromboxane synthase inhibitor, prevented these IFN $\alpha$-mediated pulmonary artery changes. This study suggests that the thromboxane cascade, a mediator of inflammation, is directly involved in the effect of IFN on the lungs [22] and may be a mediator in the development of pulmonary hypertension.

$\mathrm{HCV}$ itself has been reported to cause an occult inflammatory reaction in pulmonary air spaces. Idilman et al. [13] reported elevated levels of polymorphonuclear neutrophils in the bronchoalveolar lavage specimens of individuals with $\mathrm{HCV}$. This suggests that a low-grade chronic inflammatory reaction may be present in the lungs of individuals with $\mathrm{HCV}$ infection and may contribute to the alveolar and pulmonary arterial changes. Shiomi et al. further demonstrated that IFN $\alpha$ exacerbates this inflammatory reaction in patients with HCV. Using gallium 67 citrate scans, they evaluated patients with $\mathrm{HCV}$ before and after IFN therapy and were able to quantitatively show increased radionucleotide uptake post-treatment. These investigators concluded that IFN $\alpha$ causes a gradual subclinical pulmonary inflammatory process in a majority of the individuals treated with the agent [23].

How then might irreversible pulmonary hypertension occur in an individual receiving IFN $\alpha$ therapy? Three possible mechanisms are worth considering.

As stated earlier, IFN $\alpha$ causes an acute increase in the PVR and PAP, which results in a transient hypoxia. This results in the local release of cytokines and arachidonic acid metabolites that promote further smooth-muscle contraction, resulting in a marked transient but reversible increase in the pulmonary vascular resistance. Over a prolonged period of time, this phenomenon may promote arterial smooth-muscle hypertrophy that may lead to irreversible pulmonary artery hypertension.

A second possibility is that HCV, like HIV, may have a direct effect on the pulmonary endothelium mediated by vasoactive amines, which leads to leukocyte adherence, inflammatory cytokine release, and endothelial cell proliferation. Additionally, it has been shown that certain vasculotropic viruses, such as HHV8, play a significant etiologic role in primary pulmonary hypertension [24]. Co-infection with such viruses may yet be another etiologic factor in these individuals. This phenomenon may be subclinical initially but, with the added stress of prolonged IFN $\alpha$ exposure, may result in pulmonary hypertension which may be irreversible.

Finally, a genetic predisposition such as the novel mutation in the bone morphogenetic protein receptor type II (BMPR2) seen in certain familial forms of primary pulmonary hypertension [25] may have been present in these four cases. IFN $\alpha$ may promote the expression of this abnormal protein, which results in uncontrolled endothelial cell growth and proliferation alone or in response to insults such as HCV infection.

Regardless of the precise mechanism involved, these four cases suggest that pulmonary artery hypertension developing in individuals after prolonged IFN therapy may be irreversible and should be considered in individuals being treated with IFN $\alpha$ who present with exertional dyspnea and do not have a readily identifiable inflammatory or embolic cause for pulmonary artery hypertension. Further study is needed to identify the precise pathophysiology of this problem and the identification of individuals who might be at risk for developing this life-threatening complication of IFN $\alpha$ therapy.

Open Access This article is distributed under the terms of the Creative Commons Attribution Noncommercial License which permits any noncommercial use, distribution, and reproduction in any medium, provided the original author(s) and source are credited. 


\section{References}

1. Isaacs A, Lindenmann J. Virus interference. I. The interferon. Proc R Soc Lond B Biol Sci. 1957;147:258-267.

2. Fried MW, Shiffman ML, Reddy KR, et al. Peginterferon alfa-2a plus ribavirin for chronic hepatitis $\mathrm{C}$ virus infection. $N$ Engl $J$ Med. 2002;347:975-982.

3. Hochhaus A, Reiter A, Saußele S, et al. Molecular heterogeneity in complete cytogenetic responders after interferon-alpha therapy for chronic myelogenous leukemia: low levels of minimal residual disease are associated with continuing remission. Blood. 2000;95:62-66

4. Guilhot F, Chastang $\mathrm{C}$, Michallet $\mathrm{M}$, et al. Interferon alfa-2b combined with cytarabine versus interferon alone in chronic myelogenous leukemia. N Engl J Med. 1997;337:223-229.

5. Conlon KC, Urba WJ, Smith JW 2nd, et al. Exacerbation of symptoms of autoimmune disease in patients receiving alphainterferon therapy. Cancer. 1990;65:2237-2242.

6. Anderson P, Höglund M, Rödjer S. Pulmonary side effects of interferon-alpha therapy in patients with hematological malignancies. Am J Hematol. 2003;73:54-58.

7. Fruehauf S, Steiger S, Topaly J, et al. Pulmonary artery hypertension during interferon-alpha therapy for chronic myelogenous leukemia. Ann Hematol. 2001;80:308-310.

8. Bacon BR, Shiffman ML, Mendes F, et al. Retreating chronic hepatitis $C$ with daily interferon alfacon-1/ribavirin after nonresponse to pegylated interferon/ribavirin: DIRECT results. Hepatology. 2009;49:1838-1846.

9. Naschitz JE, Slobodin G, Lewis RJ, et al. Heart diseases affecting the liver and liver diseases affecting the heart. Am Heart J. 2000;140(1):111-120.

10. McDonnell PJ, Toye PA, Hutchins GM. Primary pulmonary hypertension and cirrhosis: are they related? Am Rev Respir Dis. 1983;127:437-441.

11. Ueda T, Ohta K, Suzuki N, et al. Idiopathic pulmonary fibrosis and high prevalence of serum antibodies to hepatitis $\mathrm{C}$ virus. Am Rev Respir Dis. 1992;146:266-268.

12. Irving WL, Day S, Johnston ID. Idiopathic pulmonary fibrosis and hepatitis C virus infection. Am Rev Respir Dis. 1993;148 (6 Pt 1):1683-1684.
13. Idilman R, Çetinkaya H, Savaş I, et al. Bronchoalveolar lavage fluid analysis in individuals with chronic hepatitis C. J Med Virol. 2001;66(1):34-39.

14. Matsumori A, Matoba Y, Sasayama S. Dilated cardiomyopathy associated with hepatitis C virus infection. Circulation. 1995;92: 2519-2525.

15. Bini EJ, Weinshel EH. Severe exacerbation of asthma: a new side effect of interferon-alpha in patients with asthma and chronic hepatitis C. Mayo Clin Proc. 1999;74:367-370.

16. Takeda A, Ikegame K, Kimura Y, et al. Pleural effusion during interferon treatment for chronic hepatitis C. Hepatogastroenterology. 2000;47:1431-1435.

17. Pietropaoli A, Modrak J, Utell M. Interferon-alpha therapy associated with the development of sarcoidosis. Chest. 1999;116: 569-572.

18. Ogata K, Koga T, Yagawa K. Interferon-related bronchiolitis obliterans organizing pneumonia. Chest. 1994;106:612-613.

19. Abi-Nassif S, Mark EJ, Fogel RB, et al. Pegylated interferon and ribavirin-induced interstitial pneumonitis with ARDS. Chest. 2003;124:406-410.

20. Karim A, Ahmed S, Khan A, et al. Interstitial pneumonitis in a patient treated with alpha-interferon and ribavirin for hepatitis $\mathrm{C}$ infection. Am J Med Sci. 2001;322(4):233-235.

21. Nakamura F, Andoh A, Minamiguchi H, et al. A case of interstitial pneumonitis associated with natural alpha-interferon therapy for myelofibrosis. Acta Haematol. 1997;97:222-224.

22. Hanaoka M, Kubo K, Hayano T, et al. Interferon-alpha elevates pulmonary blood pressure in sheep-the role of thromboxane cascade. Eur J Pharmacol. 1999;370:145-151.

23. Shiomi S, Kuroki T, Yokogawa T, et al. Changes in results of Gallium-67-citrate scanning after interferon therapy for chronic hepatitis C. J Nucl Med. 1997;38(2):216-219.

24. Cool CD, Rai PR, Yeager ME, et al. Expression of human herpesvirus 8 in primary pulmonary hypertension. $N$ Engl $\mathrm{J}$ Med. 2003;349:1113-1122.

25. International PPH Consortium, Lane KB, Machado RD, Pauciulo MW, et al. Heterozygous germline mutations in BMPR2, encoding a TGF-beta receptor, cause familial primary pulmonary hypertension. Nat Genet. 2000;26:81-84. 